\title{
The Economy of Greece and the FIFA Ranking of its National Football Team
}

\author{
By Gregory T. Papanikos*
}

\begin{abstract}
The purpose of this study is to compare the performance of the Greek economy with the FIFA ranking of the Greek National Football Team in order to find out whether there exists some sort of statistical association. The period under consideration starts with the establishment of the European and Monetary Union in 1992 and ends with the current year of 2021. In 1992, FIFA started to rank national football teams which restricts the extent of time to be used in this study. The descriptive evidence presented in this paper shows that there exists strong positive association between the level of real Gross Domestic Product (GDP) of Greece and the ranking of its national football team.
\end{abstract}

Keywords: FIFA, Greece, Football, GDP, European Union, National Teams.

\section{Introduction}

Most Greeks would agree that 2004 was a year to be remembered by Greece's current and future generations. It was an exceptional year. The Greek economy was booming, and benefited from its full membership in the Eurozone; a process which started much earlier in 1992 and was completed by the adoption of the Euro in 2002. In the beginning of the year of 2004, the city of Athens, as well as other Greek cities, were preparing to welcome the youth of the world to celebrate, once again, the modern Olympic Games in its birthplace. Athens in the beginning of 2004 had a brand-new airport, a brand-new ring road, a brand-new metro system and many other smaller and bigger infrastructures which were built either because they were required by the International Olympic Committee (IOC), or by Greece's own initiative. New stadiums were built and the old were renovated. There was great optimism, as well as vast and prodigious expectations that things will become even better in terms of economic growth and the development of sports. These high prospects were further boosted by the unexpected startling success of the Greek National Football Team, which, against all odds, won the European Trophy, beating the national team in the final game of the hosting country, Portugal. And, since in Greece all the bad and good things come in triads, Greece won the Eurovision Song Contest in 2004. What a year 2004 was!

Many Greeks committed hubris by believing that they were Gods' chosen people. It was a classic example of an ancient Greek tragedy. Metaphysically, nemesis and punishment followed. The economy disarrayed when the Great Recession hit Greece in 2009, and as a coincidence along came the failures of the Greek National Football Team. The former is measured by the Gross Domestic

\footnotetext{
*President, Athens Institute for Education and Research, Greece; Honorary Professor of Economics, University of Sterling, UK; and Professor, MLC Ljubljana, Slovenia.
} 
Product (GDP) and the latter with the Federation Internationale de Football Association (FIFA) rankings of national football teams.

This paper relates Greece's economy with the ranking of its national football team using the FIFA ranking available since 1992, which is the starting year of this paper's analysis. The FIFA methodology of rankings has changed in 1999, in 2006 and again in 2018. This methodology is not discussed here; I have briefly examined this literature and methodology in Papanikos (2017 and 2014). In the former study, I developed an ad hoc econometric model to explain the success and failures of national football teams in the finals of the 2014 World Cup hosted by Brazil. I found that political, economic and demographic variables were able to account for most of the variation of national football performances. In the latter study, (Papanikos, 2014), I developed an econometric model (a sort-of CobbDouglas production function) to examine the Greek National Football Team performance from 1960 to 2014. It was found that the performance of the Greek national team depends on the capital-labor ratio. The higher the accumulation of capital, the better the performance by the national football team. Other variables were also found important, e.g., the experience gained by playing official games, the coach and the participation in the final rounds of the World and European Football Cups organized by FIFA and UEFA respectively. UEFA is the most important regional football federation. It organizes competitions for national teams as well as the champions league of individual teams which are allocated according to the national football leagues. On the history and organization matters of UEFA, see among many other studies such as Nunes and Valerio (2020), ZambomFerraresi (2017) and Zawadzki (2015).

This paper is descriptive and no theoretical model is developed to test the hypothesis of any association between the performance of the economy and the achievements or failures of national football team. This is primarily due to the lack of reliable data both on the performance (the FIFA ranking is not the best approximation to be used in an econometric model) and of the determinants of the performance, primarily the public finances of national football. Instead, raw data are presented and discussed which reveal some interesting trends and associations between the performance of the economy and the achievements of the Greek national team football.

The paper is organized as follows: the next section provides an overview of the Greek macroeconomic performance since 1992. From an economic point of view, this period has great interest because Greece adopted the Euro as its national currency which has had significant effects on Greek economic growth and monetary stability. This issue is not examined in this paper, but those who are interested may find my econometric analysis in Papanikos (2015) informative. In the subsequent section, the FIFA rankings of the Greek National Football Team are discussed from the first year available in 1992. The last available observation during the writing of this paper was April 2021. The national football team's performance is discussed in relation to the economy's performance is discussed in the following section. The last section provides some conclusions and a direction for future research. 


\section{The Greek Economy in the EMU Period}

In 1992 the European Union (EU) launched its biggest project ever called Economic and Monetary Union (EMU) with an objective to: ${ }^{1}$

- Coordinate economic policy-making between member states.

- Coordinate fiscal policies by limiting government debt and deficit.

- Follow an independent monetary policy run by the European Central Bank (ECB)

- Supervise financial institutions within the Euro area by applying single rules.

- Introduce a common currency to be used by all member states.

The rationale behind this deepening of economic integration was (a) higher economic growth (more jobs) and (b) monetary stability (lower inflation). The herculean task of the EMU was the introduction of a common currency-the Eurowhich came into circulation 1 January 2002 in twelve EU countries; Greece was one of them. Today, 19 of the 27 EU countries are using Euro as their national currency. In Papanikos (2015), I have examined the economic growth effects of introducing the Euro. The conclusion of the study was that the Euro was not a problem, but its over-evaluation had negative impacts on some economies, including the Greek one. This important issue goes beyond the scope of this paper.

Table 1. Greek Economic Growth, 1992-2021

(GDP is measured in billions of constant 2015 of euro)

\begin{tabular}{|l|c|c|c|c|c|c|c|c|}
\hline YEAR & GDP & GDP $(\boldsymbol{\%})$ & YEAR & GDP & $\begin{array}{c}\text { GDP } \\
(\boldsymbol{\%})\end{array}$ & YEAR & GDP & $\begin{array}{c}\text { GDP } \\
(\boldsymbol{\%})\end{array}$ \\
\hline 1992 & 148.25 & 0.70 & 2002 & 196.64 & 3.92 & 2012 & 180.56 & -7.08 \\
\hline 1993 & 145.88 & -1.60 & 2003 & 208.03 & 5.79 & 2013 & 175.61 & -2.74 \\
\hline 1994 & 148.80 & 2.00 & 2004 & 218.56 & 5.06 & 2014 & 176.84 & 0.70 \\
\hline 1995 & 151.92 & 2.10 & 2005 & 219.87 & 0.60 & 2015 & 176.11 & -0.41 \\
\hline 1996 & 156.27 & 2.86 & 2006 & 232.30 & 5.65 & 2016 & 175.25 & -0.49 \\
\hline 1997 & 163.28 & 4.48 & 2007 & 239.90 & 3.27 & 2017 & 177.49 & 1.28 \\
\hline 1998 & 169.64 & 3.89 & 2008 & 239.10 & -0.34 & 2018 & 180.26 & 1.56 \\
\hline 1999 & 174.85 & 3.07 & 2009 & 228.81 & -4.30 & 2019 & 183.61 & 1.86 \\
\hline 2000 & 181.71 & 3.92 & 2010 & 216.28 & -5.48 & 2020 & 167.09 & -9.00 \\
\hline 2001 & 189.21 & 4.13 & 2011 & 194.33 & -10.15 & 2021 & 175.48 & 5.02 \\
\hline
\end{tabular}

Source: Economic Commission (Ameco Data Base).

What was the performance of the Greek economy since 1992? Table 1 reports total and growth rates of GDP. Per capita income could have been used, but given that the population has not changed much, the analysis would have been the same.

\footnotetext{
${ }^{1}$ See the information provided in the official website of the European Commission -the governing body of the European Union- available at https://ec.europa.eu/info/business-econo my-euro/econo mic-and-fiscal-policy-coordination/economic-and-monetary-union/what-economic-and-monetaryunion-emu_en.
} 
In some cases, using population statistics may not capture the true value of the economy because of Greek outmigration due to the economic crisis of the 2010s, which underestimates the true value of per capita income when it is measured as the GDP-population ratio.

The following two figures depict, in a better way than the table, the oscillations of GDP and GDP growth during the 1992-2022 period. The last two years (2020 and 2021) are preliminary forecast values of the European Commission which is the source of the data used in this study. Figure 1 shows a much-expected uprising in GDP after the establishment of the EMU in 1992 and the introduction of the Euro in 2002. As shown in Figure 2, the Greek economy experienced a positive growth rate. But in 2008, the Greek economy was hit hard by the Great Recession leading to an unprecedented loss of output for peace years. Never before the GDP growth rate was so low, which, in 2011, marked a decline of $10.15 \%$. At the time that the Greek economy was emerging out of the recession, coupled with the negative rates of GDP growth, the pandemic hit its economy very hard, as it did all the major European countries, as I have explained in Papanikos (2021). The expected loss of output in 2020 is $9 \%$, which most probably will be realized, but there are serious doubts that the European Commission's forecast of $5.02 \%$ for 2021 will be ever achieved given the continuous negative impacts of the pandemic on the Greek economy.

Figure 1. Greek Domestic Product (GDP), 1992-2022

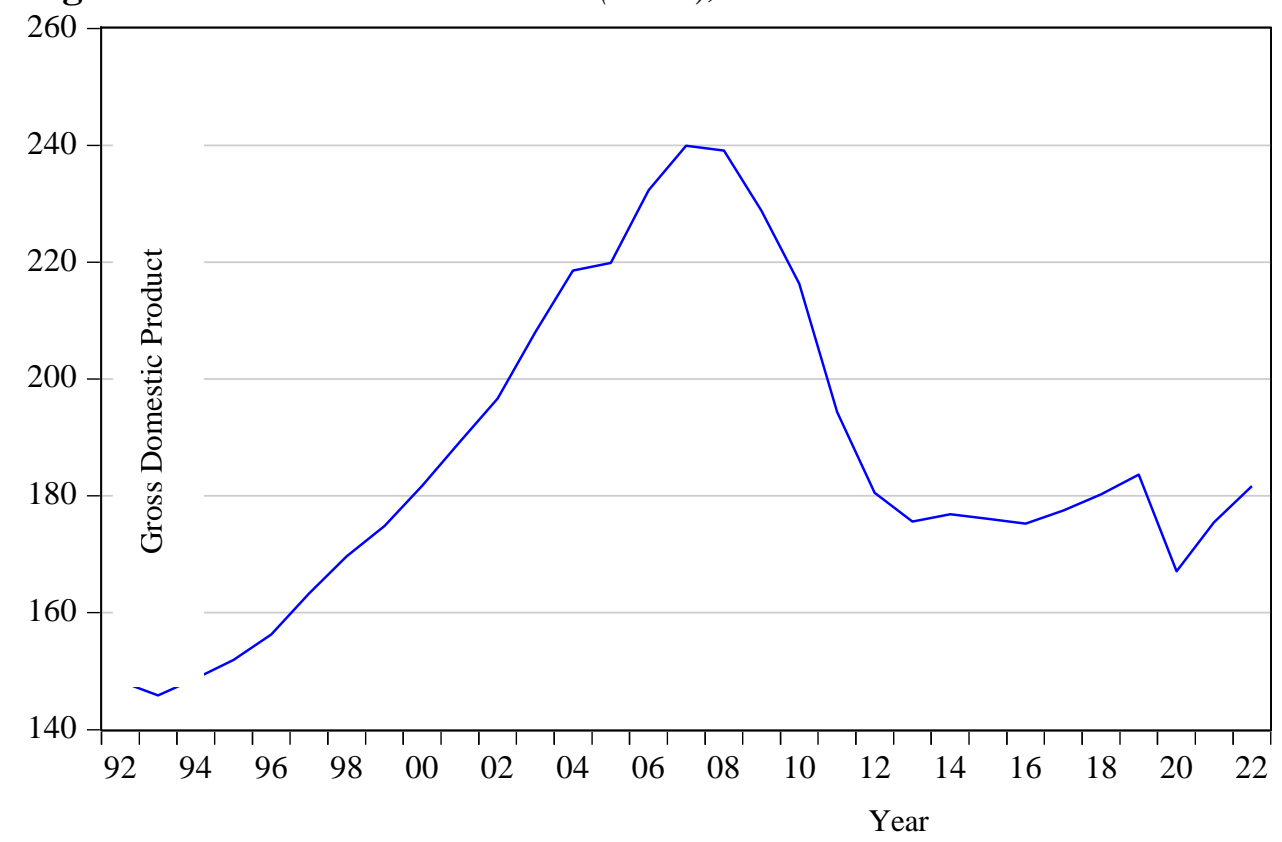


Figure 2. The Rate of Growth of Greek Domestic Product (GDP), 1992-2022

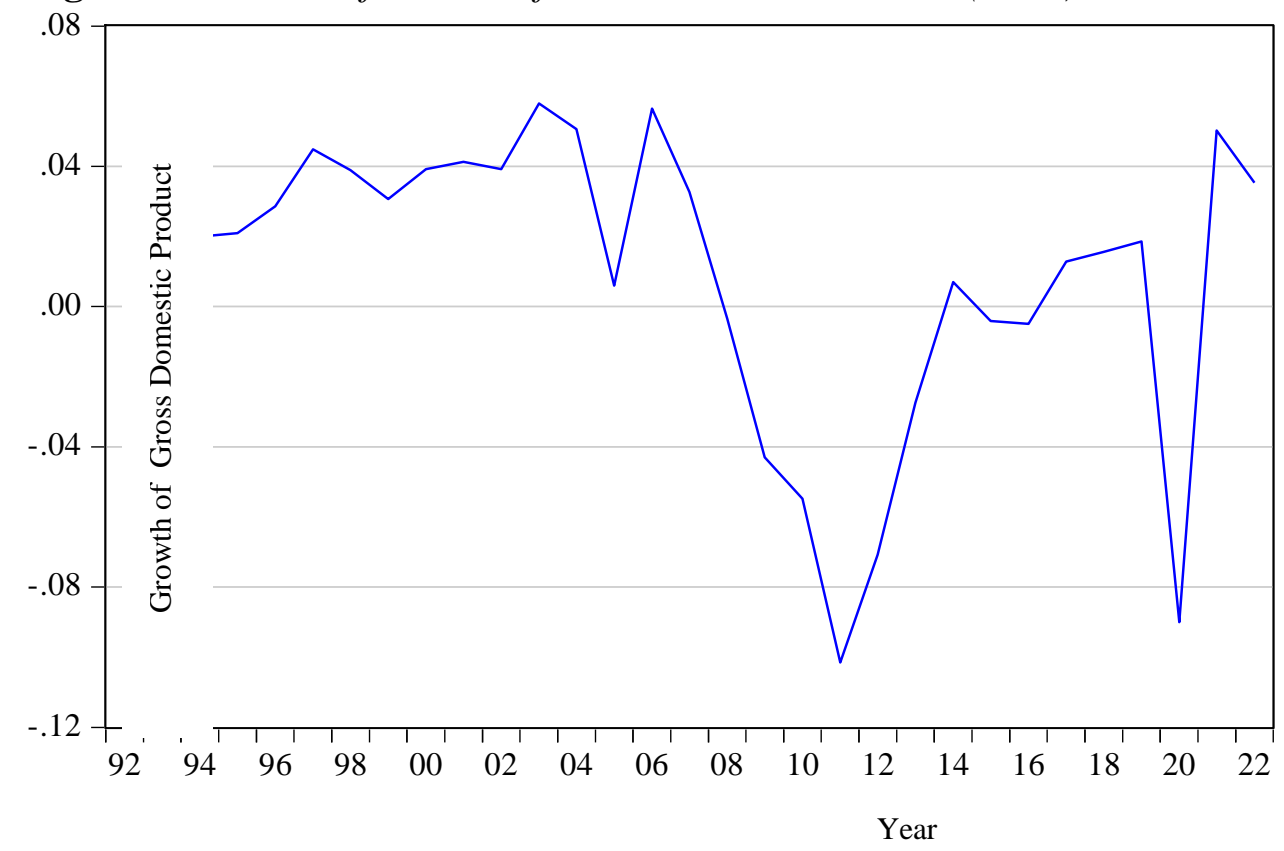

The data in Table 1 are presented in blocks of three decades, which, as by coincidence, have their own important economic phenomenology, to use a philosophical term. The first decade of the period under investigation in this paper (1992-2001) is marked by the efforts to establish the EMU and better prepare the economies to adopt a new common currency. The second decade (2002-2011) of the data set coincides with the introduction of the new currency, 1 January 2002, and, at the end of the decade, with the beginning of the so-called Great Recession. The next decade (2012-2021) begun with the end of Great Recession, but it ended with the beginning of the pandemic effect, which its impact is expected to continue for the years to come.

Which of the three decades was better? Undoubtedly the second decade, as is shown in Table 2, which compares the summary statistics of GDP of the three tenyear periods. An important indicator is the range of difference between the maximum and the minimum value of output produced in the decade. During the 2002-2011 period, the Greek economy produced an extra 45.57 billion euro if the maximum and the minimum GDP of the period are compared. The decade's average GDP was 219.38 billion of constant 2015 euro much higher than the firstand third-decade's average of 162.98 and 176.83 billion respectively. The last three measures are also important indicators of the higher moments of the GDP distribution showing that there were statistically significant differences between the three decades. 
Table 2. Summary Statistics of Greek GDP, 1992-2021

\begin{tabular}{|l|c|c|c|c|}
\hline Statistic & $\mathbf{1 9 9 2 - 2 0 2 1}$ & $\mathbf{1 9 9 2 - 2 0 0 1}$ & $\mathbf{2 0 0 2 - 2 0 1 1}$ & $\mathbf{2 0 1 2 - 2 0 2 1}$ \\
\hline Average & 186 & 162.98 & 219.38 & 176.83 \\
\hline Minimum & 146 & 145.88 & 194.33 & 167.09 \\
\hline Maximum & 239 & 189.21 & 239.90 & 183.61 \\
\hline Range & 93 & 43.33 & 45.57 & 16.52 \\
\hline Standard Deviation & 27 & 15.28 & 16.16 & 4.39 \\
\hline Skewness & 0.54 & 0.45 & -0.27 & -0.73 \\
\hline Kurtosis & 2.37 & 1.83 & 1.89 & 3.82 \\
\hline
\end{tabular}

Recapitulating this section's discussion, any student of economics would expect the economy to be subject to economic cycles. Nothing remains stable in the economy; there are ups and downs, and good and bad years. The 1992-2021 period of the Greek GDP cycles constitute a textbook example. The economy experienced an expansion in the 1994-2008 period pulling the economy out of its tough years of 1992-1993. It reached a GDP peak in 2008 of 239 billion euro before the economy started to contract again. Just before it started to recover, the economy was hit by the negative unexpected shock of the pandemic, which had and still has devastating economic (measured in output loss) and non-economic effects (measured in life losses). Many economists expect that once the pandemic is over, the Greek economy would be able to return to its long-term positive economic growth rates.

\section{FIFA Ranking of the Greek National Football Team}

FIFA reports its football rankings of national teams every month. All data reported and analyzed here are end-of-year (December) rankings. The number reported in Table 3 is the ranking of the Greek National Football Team relative to all national teams which are members of FIFA. In order to increase its rank, the Greek team must outperform the other teams. Table 4 reports summary statistics for the total dataset, as well as for the three decades separately.

Similar to the economy's performance, the best decade is the second of the dataset, 2002-2011. The average position of this period was 20 (the Greek National Football Team was ranked in the $20^{\text {th }}$ place, meaning that 19 teams were ranked higher and all the others -more than 100 teams-, below) which is considerably different from the other two decades, the first and the third, which both had a similar average ranking of 39 and 38 respectively. In two years of the second decade (2007 and 2010), Greece reached the highest ever rank of $11^{\text {th }}$ place in the world, which was also achieved in the first year (2012) of the third ten-year period. However, during the last decade, the Greek National Football Team lost 43 positions in the FIFA ranking from the $11^{\text {th }}$ place in 2012 to the $54^{\text {th }}$ in 2019. 
Table 3. FIFA Rank of the Greek National Football Team

\begin{tabular}{|l|c|c|c|c|c|}
\hline YEAR & FIFA RANK & YEAR & $\begin{array}{c}\text { FIFA } \\
\text { RANK }\end{array}$ & YEAR & $\begin{array}{c}\text { FIFA } \\
\text { RANK }\end{array}$ \\
\hline 1992 & 31 & 2002 & 48 & 2012 & 11 \\
\hline 1993 & 34 & 2003 & 30 & 2013 & 12 \\
\hline 1994 & 28 & 2004 & 18 & 2014 & 24 \\
\hline 1995 & 34 & 2005 & 16 & 2015 & 41 \\
\hline 1996 & 35 & 2006 & 16 & 2016 & 42 \\
\hline 1997 & 42 & 2007 & 11 & 2017 & 47 \\
\hline 1998 & 53 & 2008 & 20 & 2018 & 43 \\
\hline 1999 & 34 & 2009 & 13 & 2019 & 54 \\
\hline 2000 & 42 & 2010 & 11 & 2020 & 53 \\
\hline 2001 & 57 & 2011 & 14 & 2021 & 51 \\
\hline
\end{tabular}

Table 4. Summary Statistics of FIFA Ranking

\begin{tabular}{|l|c|c|c|c|}
\hline Statistic & $\mathbf{1 9 9 2 - 2 0 2 1}$ & $\mathbf{1 9 9 2 - 2 0 0 1}$ & $\mathbf{2 0 0 2 - 2 0 1 1}$ & $\mathbf{2 0 1 2 - 2 0 2 1}$ \\
\hline Average & 32 & 39 & 20 & 38 \\
\hline \multirow{2}{*}{ Worse Position } & 57 & 57 & 48 & 54 \\
& $(2001)$ & $(2001)$ & $(2002)$ & $(2019)$ \\
\hline \multirow{2}{*}{ Best Position } & 11 & 31 & 11 & 11 \\
& $(2007,2010,2012)$ & $(1992)$ & $(2007,2010)$ & $(2012)$ \\
\hline \multirow{2}{*}{ Range } & 46 & 26 & 37 & 43 \\
& $(57-11)$ & $(57-31)$ & $(48-11)$ & $(54-11)$ \\
\hline Standard Deviation & 15.2 & 9.5 & 11.4 & 16.27 \\
\hline Skewness & -0.01 & 0.86 & 1.74 & -0.76 \\
\hline Kurtosis & 1.67 & 2.48 & 4.91 & 2.04 \\
\hline
\end{tabular}

Of interest are the differences in the standard deviation (showing stability), as well as the ones of the higher moments of the rank distribution of skewness (showing symmetry of the distribution) and kurtosis (showing thickness). Ideally, a team aims to not only achieve high rank, but at the same time stay there for a long period, i.e., minimize the standard deviation. This was not achieved by the Greek National Football Team in any of the three decades under consideration. The standard deviation was high, something which is also indicated by the high range of the positions in each decade. The Greek team is characterized by being relatively unstable in its performance.

This instability is better shown in Figure 3 which graphs the inverse of the FIFA ranking. The reported data in Figure 3 are transformed values of the FIFA ranking, by being divided by 100 , by the position of the Greek National Football Team according to FIFA ranking. For example, the $11^{\text {th }}$ position in the FIFA ranking takes the value of close to 10 which is the higher value of this transformed presentation. On the other hand, the $50^{\text {th }}$ position will take the value of 2 . This is a better way of presenting because an upward move along the curve is interpreted as an improvement. 
Figure 3. The FIFA Ranking of the Greek National Football Team, 1992-2021

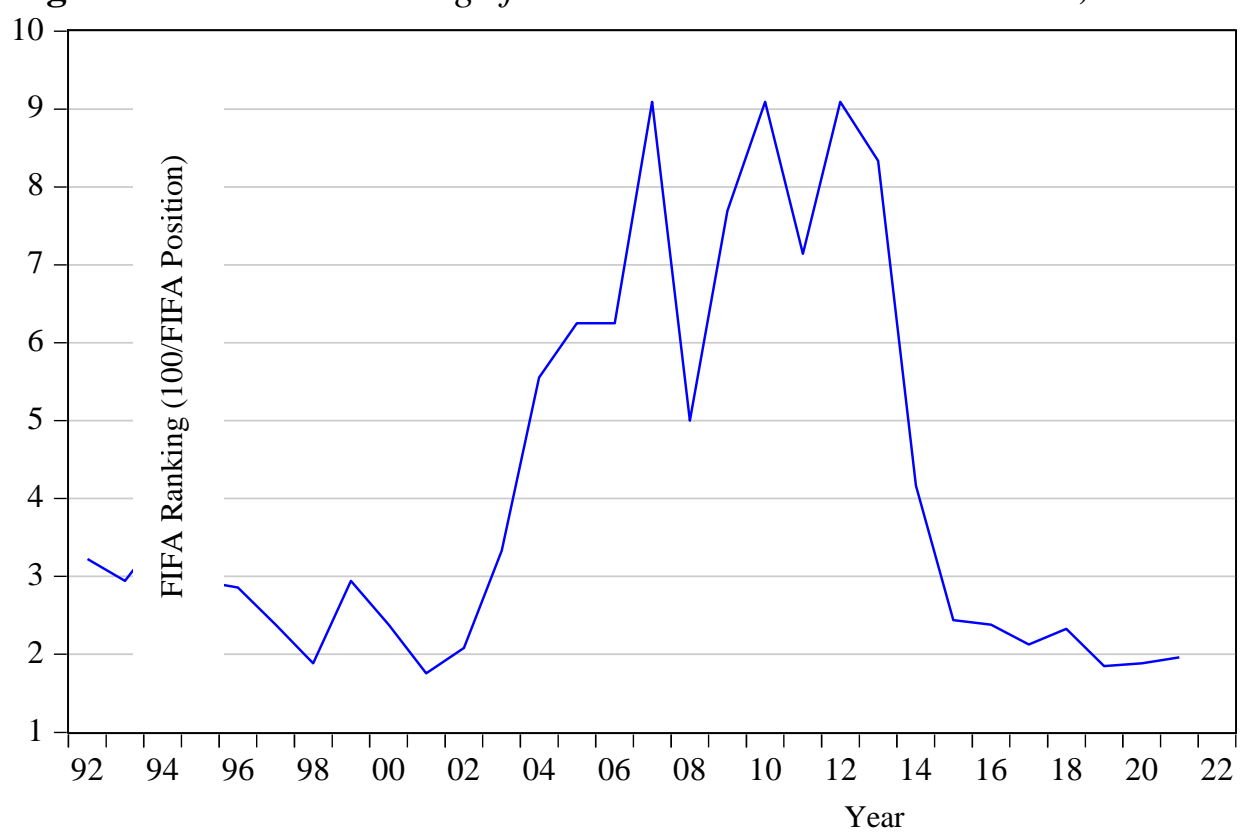

Figure 3 shows remarkable differences between the three decades which are similar, but not identical, to what was observed in the previous section for GDP. During the first decade, the ranking remained relatively stable, which is also demonstrated with the low range and standard deviation of this decade, relative of the other two decades (see Table 4). The next decade (2002-2011) is the golden period of Greek football performance. In 2004, Greece won the European Trophy; an achievement to be remembered for many years to come. In the last decade, the rankings of the Greek National Football Team are declining.

Summarizing this section, the FIFA ranking of the Greek National Football Team shows great variations. Its best decade was the second one (2002-2011), which, as a coincidence, overlaps with the best performance of the Greek economy. One is then prompted to ask the question whether the two (the economy and football performances) are somehow related. This question is addressed in the next section of the paper.

\section{The Association between the Economy and National Football Performance}

Many economists and non-economists have argued against the use of GDP as a measure of national or social welfare. Nobody disagrees that the index of GDP is not a perfect measure, and better measures can be envisaged which better account for what one may call national welfare or happiness or pride or eudaimonia.

The difficulty, however, lies on the fact that these other "measures" cannot be measured, i.e., they cannot be expressed in quantitative terms. Even worse, people's definition of national welfare differs. All these difficulties may be surpassed if we assume that the GDP is a measure not only of the total number of goods and services produced in a country in a given year only, but it might be a 
good index of the eudaimonia in a society. When a nation or society is doing very well in terms of its culture, sports and other spiritual activities, then its economy might also do well. In a happy society, where every citizen who is proud to belong to such a nation, has an incentive to work harder and produce more output, avoiding all activities which undermine the economic growth of the country. Euphoria and optimism are important determinants of private investment and therefore economic growth. In that sense, I consider success in sports and culture as a determinant of economic growth.

But it works the other way as well. When the economy is booming, more resources can be diverted into sports and cultural activities, and therefore creates the necessary conditions for better national performance in such activities. For these reasons, it is very difficult to draw a deterministic causal model of the relationship between sports and the economy, or in general, culture (which includes sports) and the economy. We can only talk about positive or negative associations (correlations). In this section of the paper, I look at one such association, namely the performance of the national football team and economic growth. If the above arguments have any validity, this association should be positive.

In the previous two sections, the graphics and the raw data hinted that there might be a positive association or relation between GDP and the performance of the Greek National Football Team. Separating the sample into three decades, it becomes clear that in the last two decades the GDP and the football performance have moved in parallel. Only in the first decade it appears that there was no relation. The economy was doing relatively well, but football's performance was relatively stable.

Figure 4 reports the scatter diagram of FIFA rankings and the GDP level for the entire 1992-2021 period. Also, a regression line has been inserted which better depicts the average association between the two variables.

Figure 4 clearly depicts a strong positive association of the FIFA ranking and the level of GDP. It appears that when the economy is doing well, the Greek football at the national level is also doing well. The correlation coefficient between the two variables was +0.577 . Using a simple logarithmic regression specification, it was found that a $10 \%$ increase in GDP results to a $2 \%$ climb in the FIFA ranking.

Figure 4 also reveals that the exceptional positions of the $11^{\text {th }}$ place in the FIFA ranking in the three years mentioned above $(2007,2010,2012)$ appear in the graph as outliers along with the $12^{\text {th }}$ position achieved in 2013 . For some reasons, these years showed there was an overshooting in the performance of the Greek National Football Team reflecting in part the legacy of the great success of 2004 when the Greek National Football Team won the European Trophy. After all, the beauty of the game is that sometimes the underdogs may perform very well. If outliers are excluded then the association between the economy and the national football performance becomes stronger. The correlations coefficient from +0.577 increases to +0.6503 . 
Figure 4. The Association of the GDP and the FIFA Ranking, 1992-2022

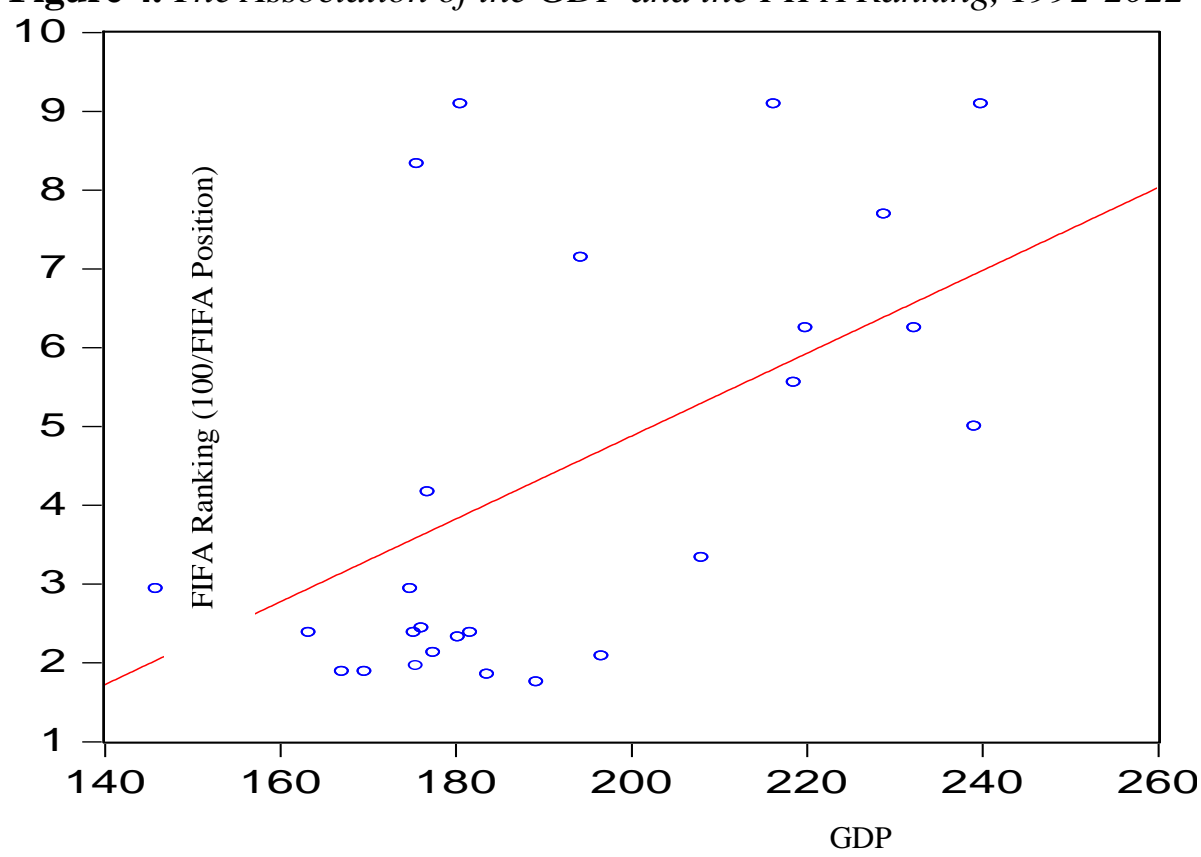

The above analysis does not show causation and the relation might be purely spurious. However, even though arguments could be made that the causality may run both ways, the causality from the economy to football has a solid theoretical foundation. After all, football is an expensive leisure and cultural activity, and can be satisfied better if the economy is able to allocate public funds. The richer the economy (the higher the GDP), the higher the funds available for sports (football) development, and therefore the higher the performance of its national football team. Even exceptions do exist; on average, rich countries do have better performing national football teams as I demonstrated in my other study (Papanikos, 2017).

\section{Conclusions}

There appears to be a strong association between the performance of the economy and the achievements of the national football team. A $10 \%$ increase in Greece's GDP is associated with an increase of $2 \%$ in the FIFA ranking of its national football team. The correlation coefficient is +0.577 for the entire period, but if we exclude the outliers in performance then the correlation coefficient increases to +0.6503 .

The entire period of 1992 to 2021 was divided into three ten-year periods. It appears that with the exception of the first decade, the football performance was associated with the performance of the economy. A difference is observed in the first decade (1992-2001) during which the performance of the Greek team remained stable when the economy was increasing.

The purpose of the study was not to draw conclusions as of the causal relationship between the two variables. Theoretically, the causality may run both 
ways even though the most probable one is the economy to affect the national football performance. This requires further research, but the descriptive (graphical) depiction of the data show that the national football performance usually follows the previous years' economic growth performance with a lag. Testing for causality is left for a future research.

\section{References}

FIFA (2021) Revision of the FIFA/Coca-Cola world ranking. Retrieved from: https://img. fifa.com/image/upload/edbm045h0udbwkqew35a.pdf. [Accessed 15 April 2021]

Nunes AB, Valério N (2020) UEFA: a successful pan-European organization during the cold war. Athens Journal of Sports 7(1): 55-76. DOI=10.30958/ajspo.7-1-4.

Papanikos GT (2014) An econometric evaluation of the performance of the Greek national football team. Athens Journal of Sports 1(4): 233-246. DOI=10.30958/ajspo.1-4-3.

Papanikos GT (2015) The real exchange rate of Euro and Greek economic growth. The Journal of Economic Asymmetries 12(2): 100-109.

Papanikos GT (2017) Economic, population and political determinants of the 2014 World Cup match results. Soccer \& Society 18(4): 516-532. DOI=10.1080/14660970.2015. 1067799.

Papanikos GT (2021) The European Union's recovery plan: a critical evaluation. Athens Journal of Mediterranean Studies 7(2): 85-102. DOI=10.30958/ajms.7-2-1.

Zambom-Ferraresi F, García-Cebrián LI, Lera-López F (2017) Sports results measurement and efficiency in UEFA Champions League. Athens Journal of Sports 4(4): 291-312. DOI=10.30958/ajspo.4.4.4.

Zawadzki K (2015) Euro 2012 in Gdansk, Poland. Is it worth using public funds? Athens Journal of Sports 2(2): 85-98. DOI=10.30958/ajspo.2-2-2. 
